\title{
A comparative study of postoperative outcomes after stapled versus handsewn gastrojejunal anastomosis for pylorus-resecting pancreaticoduodenectomy
}

\author{
Sook Hyun Lee ${ }^{1}$, Yun Ho Lee ${ }^{2}$, Young Hoe Hur ${ }^{2}$, Hee Joon Kim ${ }^{1}$, and Byung Gwan Choi ${ }^{2}$
}

\author{
${ }^{1}$ Department of Surgery, Chonnam National University Hospital and Medical School, Gwangju, \\ ${ }^{2}$ Department of Surgery, Chonnam National University Hwasun Hospital and Medical School, Hwasun, Korea
}

\begin{abstract}
Backgrounds/Aims: A stapler is widely used in various surgeries, and there have been recent attempts to use it for performing duodenojejunostomy and gastrojejunostomy during pancreaticoduodenectomy. This study aimed to compare the postoperative results of handsewn gastrojejunostomy (HGJ) and stapled gastrojejunstomy (SGJ) limited to pylorus-resecting pancreaticoduodenectomy (PrPD) performed by a single surgeon. Methods: This retrospective study was conducted between January 2014 and March 2020, and included 131 patients who underwent PrPD performed by a single surgeon. Of the total subjects, 90 were in the HGJ group and 41 in the SGJ group. Results: The mean time of surgery was significantly shorter in the stapled group than in the handsewn group $(450.4 \pm 75.4 \mathrm{~min}$ vs. $397.1 \pm 66.5$ $\min , p<0.001)$. However, there were no significant differences between the groups in the rates of postoperative pancreatic fistula, bile leak, chyle leak, intra-abdominal fluid collection, postoperative bleeding, ileus, Clavien-Dindo, rate of reoperation, and 30-day mortality, including delayed gastric emptying (DGE) ( $\mathrm{n}=11$ vs. $\mathrm{n}=6, p=0.92$ ). Conclusions: Gastrojejunostomy using a stapler in PrPD reduces the reconstruction time without any increase in the rate of complications, including DGE. Therefore, using a stapler for gastrojejunostomy in pancreaticoduodenectomy is feasible and safe. (Ann Hepatobiliary Pancreat Surg 2021;25:84-89)
\end{abstract}

Key Words: PrPD; DGE; Stapler; Pancreaticoduodenectomy; Single surgeon

\section{INTRODUCTION}

Pancreaticoduodenectomy is the standard surgical method for benign and malignant tumors of the pancreatic head or periampullary region. ${ }^{1}$ However, compared to the other abdominal surgeries, complications such as anastomotic leakage, stricture, bleeding, dumping syndrome, and delayed gastric emptying are more likely to occur in this surgery due to various anastomoses created for reconstruction after resection. ${ }^{2,3}$ Patients with many underlying diseases might develop medical or systemic complications due to the long operation time. ${ }^{4,5}$ With the development of surgical tools and surgical methods, stapling has been used for intestinal anastomosis in several surgeries to ease the complex surgical process and to reduce the time of surgery compared to that in manual anastomosis. ${ }^{6-9}$ After pancreaticoduodenectomy, the stapler has also been used for duodenojejunostomy or gastrojejunostomy, and it has been reported that operation time was reduced. Moreover, the incidence of delayed gastric emptying (DGE) after surgery decreased in patients who underwent gastro/ duodenojejunostomy using stapling anastomosis after pylorus-preserving pancreticoduodenectomy (PPPD) and Whipple's operation. ${ }^{10}$ However, some reports also mentioned that the decrease in the incidence of DGE was not significant. ${ }^{11}$ The above studies analyzed several surgical methods such as Whipple's op, PPPD, and pylorus-resecting pancreaticoduodenectomy (PrPD); therefore, factors other than the handsewn and stapled anastomosis might have influenced the outcomes. This study aimed to compare the postoperative results of stapled gastrojejunostomy and handsewn gastrojejunostomy in patients who under-

Received: September 24, 2020; Revised: October 14, 2020; Accepted: October 15, 2020

Corresponding author: Young Hoe Hur

Department of Surgery, Chonnam National University Hwasun Hospital and Medical School, 322 Seoyang-ro, Hwasun 58128, Korea Tel: +82-61-379-7646, Fax: +82-61-379-7661, E-mail: surgihur@naver.com

Copyright (C) 2021 by The Korean Association of Hepato-Biliary-Pancreatic Surgery

This is an Open Access article distributed under the terms of the Creative Commons Attribution Non-Commercial License (http://creativecommons.org/ licenses/by-nc/4.0) which permits unrestricted non-commercial use, distribution, and reproduction in any medium, provided the original work is properly cited. Annals of Hepato-Biliary-Pancreatic Surgery • pISSN: 2508-5778 - eISSN: 2508-5859 
sent PrPD performed by a single surgeon.

\section{MATERIALS AND METHODS}

\section{Patients population}

We reviewed the data of 211 patients in whom pancreaticoduodenectomy was planned as an elective surgery between January 2014 and to March 2020. Nine patients who underwent PPPD, 27 patients who underwent Whipple's operation, and 44 patients with distant lymph node metastasis, liver metastasis, angioinvasion, or those who underwent palliative surgeries were excluded. Finally, 131 patients who underwent PrPD were enrolled; 90 patients underwent handsewn gastrojejunostomy (HGJ), and 41 underwent stapled gastrojejunostomy (SGJ). This study was approved by the Institutional Review Board of Hwasun Chonnam National University Hospital (IRB No. CNUHH2020-144).

\section{Surgical procedure}

All surgeries were performed under laparotomy. The stomach was incised $2 \mathrm{~cm}$ proximal to the pylorus, so that the antrum and the entire stomach was preserved. The gallbladder, pancreatic head with the bile duct, pylorus, and proximal jejunal loop were excised. Lymphadenectomy to similar extent was performed in all patients. Pancreaticojejunostomy was performed in an end-to-side, duct-tomucosa anastomosis. Hepaticojejunostomy was performed $15 \mathrm{~cm}$ distal to the pancreaticojejunostomy site. For gastrojejunostomy, antecolic Billroth II reconstruction was performed distal to the hepaticojejunostomy site without Braun anastomosis. In the HGJ group, gastrojejunostomy was performed with an end-to-side anastomosis between the rest of the stomach and the jejunum. The mucosa was sewn with a continuous sutured using Vicryl Plus ${ }^{\circledR} 3 / 0$, and the seromuscular layer was sewn with an interrupted suture. The SGJ group was underwent side-to-side anastomosis between the greater curvature of the stomach and the jejunum using a linear stapler $75 \mathrm{~mm}$ (ETHICON Linear Cuter NTLC75, Johnson \& Johnson). The opening length of the anastomosis was $60 \mathrm{~mm}$. In both groups, interrupted seromuscular Lambert sutures for the outer layer were performed with Sofsilk ${ }^{\circledR} 3 / 0$. After reconstruction, a drainage tube was inserted into the pancreaticojejunostomy and hepaticojejunostomy site.

\section{Postoperative management}

The nasogastric tube (NGT) was inserted before surgery in all patients and was removed on postoperative day (POD) 1 if the drainage volume was below $200 \mathrm{ml} /$ day and not blood stained. Oral intake was initiated by providing sips of water after removing the NGT. A soft diet was started on POD 3. If vomiting or severe distention of the stomach was observed on abdominal radiography after surgery, the NGT was reinserted. The amylase levels in the serum and in thedrain fluid were measured on or after POD 1, 3, and 7. Prophylactic octreotide (Sandostatine ${ }^{\circledR}$, $100 \mu \mathrm{g}$ subcutaneously three times per day, Novartis, Rueil Malmaison, France) was administered routinely to all patients for 7 days from the day before surgery. Proton pump inhibitors were injected in all patients after surgery, and were switched to oral medications after the initiation of diet, and administered until 30 days after discharge. Abdominal computed tomography was performed on POD 7 , and the abdominal drain was removed on the same day if there were no signs of postoperative pancreatic fistula (POPF), intra-abdominal collection, or other complications related to the surgery.

\section{Definitions of outcome measures}

According to the International Study Group of Pancreatic Surgery, DGE was defined as the need for or re-insertion of NGT after POD 3 or failure to start oral diet on POD 7. The DGE was subdivided into grades $\mathrm{A}, \mathrm{B}$, and $\mathrm{C}$ in the order of increasing severity. Grade A was defined as the need for NGT within POD 4-7, reinsertion of NGT after removal at POD 3, or inability to tolerate a solid diet on POD 7. Grade B was defined as the need for NGT from POD 8 to 14, reinsertion of the NGT after POD 7, or inability to tolerate a solid diet on POD 14. Grade C was defined as the inability to remove the NGT, reinsertion of NGT after POD 14, or inability to tolerate a solid diet on POD $21 .^{12}$

POPF was defined according to the guidelines of the International Study Group on Pancreatic Fistula (ISGPF) as an amylase level in the drain fluid more than three times that of the serum amylase on or beyond POD 3. POPF grades were stratified based on the revised ISGPF definition in 2016. Patients were assigned POPF grades $\mathrm{B}$ and $\mathrm{C}$ if they had a clinically relevant condition related directly to POPF, and POPF grade A if there was no 
change in their clinical condition, or had a "biochemical leak". 13

\section{Statistical analysis}

Statistical analysis was performed using SPSS version 22.0 (SPSS Inc., Chicago, IL, USA). Student's $t$-test or Mann-Whitney $U$ test were used to compare the continuous variables, and Fisher's exact test or chi-square test were used to compare the categorical variables. All $p$-values were two-sided, and $p<0.05$ was considered significant.

\section{RESULTS}

A total of 131 patients underwent PrPD; 90 in the HGJ group and 41 in the SGJ group. The relevant patient characteristics and perioperative details are shown in Table 1. There were no significant differences in the age, sex, BMI, American Society of Anesthesiologists (ASA) score $\geq 3$, underlying diseases such as hypertension and diabetes mellitus, previous operation history, preoperative total bilirubin or preoperative bile drainage between the groups. Histologically, bile duct carcinoma was the most common type of tumor in both groups ( $\mathrm{n}=28$ vs. $\mathrm{n}=17$, $p=0.338$ ). Further, no significant difference in pancreatitis

Table 1. Patient and operative characteristics

\begin{tabular}{|c|c|c|c|}
\hline Variables & Handsewn $(n=90)$ & Stapled $(n=41)$ & $p$ \\
\hline Age & $66.6 \pm 11.6$ & $66.7 \pm 9.5$ & 0.966 \\
\hline Sex & & & 0.403 \\
\hline Male & $44(48.9 \%)$ & $25(58.5 \%)$ & \\
\hline Female & $46(51.1 \%)$ & $17(41.5 \%)$ & \\
\hline BMI & $23.0 \pm 3.2$ & $22.7 \pm 3.1$ & 0.675 \\
\hline ASA classification & & & 0.059 \\
\hline$<3$ & $76(84.4 \%)$ & $28(68.3 \%)$ & \\
\hline$\geq 3$ & $14(15.6 \%)$ & $13(31.7 \%)$ & \\
\hline \multicolumn{4}{|l|}{ Underlying disease } \\
\hline HTN & $50(55.6 \%)$ & $23(56.1 \%)$ & 1.000 \\
\hline $\mathrm{DM}$ & $36(40.0 \%)$ & $10(24.4 \%)$ & 0.124 \\
\hline Heart & $11(12.2 \%)$ & $6(14.6 \%)$ & 0.920 \\
\hline Pulmonary & $4(4.4 \%)$ & $2(4.9 \%)$ & 1.000 \\
\hline Hepatic & $3(3.3 \%)$ & $2(4.9 \%)$ & 1.000 \\
\hline Renal & $2(2.2 \%)$ & $0(0.0 \%)$ & 0.847 \\
\hline Cerebrovascular & $5(5.6 \%)$ & $4(4.9 \%)$ & 0.611 \\
\hline Previous operation history & $17(18.9 \%)$ & $12(29.3 \%)$ & 0.271 \\
\hline Preoperative total bilirubin & $1.6 \pm 1.9$ & $1.4 \pm 1.8$ & 0.477 \\
\hline Preoperative bile drainage (ENBD, ERBD, PTBD) & $50(56.6 \%)$ & $26(63.4 \%)$ & 0.412 \\
\hline \multicolumn{4}{|l|}{ Tumor type } \\
\hline Pancreatic adenocarcinoma & $17(18.9 \%)$ & $2(4.9 \%)$ & 0.065 \\
\hline Bile duct carcinoma & $28(31.1 \%)$ & $17(41.5 \%)$ & 0.338 \\
\hline Ampullary adenocarcinoma & $22(24.4 \%)$ & $7(17.1 \%)$ & 0.474 \\
\hline Duodenal adenocarcinoma & $1(1.1 \%)$ & $2(4.9 \%)$ & 0.480 \\
\hline Intraductal papillary neoplasms & $12(13.3 \%)$ & $7(17.1 \%)$ & 0.767 \\
\hline Pancreatic endocrine tumor & $4(4.4 \%)$ & $0(0.0 \%)$ & 0.410 \\
\hline Other diseases & $7(7.8 \%)$ & $6(14.6 \%)$ & 0.367 \\
\hline Pancreatitis & $26(28.9 \%)$ & $9(22.0 \%)$ & 0.536 \\
\hline Pancreas texture & & & 0.409 \\
\hline Soft & $60(66.7 \%)$ & $31(75.6 \%)$ & \\
\hline Hard & $30(33.3 \%)$ & $10(24.4 \%)$ & \\
\hline Vein resection (PV, SMV, IVC) & $11(11.2 \%)$ & $4(9.8 \%)$ & 0.908 \\
\hline
\end{tabular}

BMI, body mass index; ASA, American Society of Anesthesiologists; HTN, hypertension; ENBD, endoscopic nasobiliary drainage; ERBD, endoscopic retrograde biliary drainage; PTBD, percutaneous transhepatic biliary drainage; PV, portal vein; SMV, superior mesenteric vein, IVC, inferior vena cava 
Table 2. Postoperative outcomes

\begin{tabular}{lccc}
\hline \multicolumn{1}{c}{ Variables } & Handsewn $(\mathrm{n}=90)$ & Stapled $(\mathrm{n}=41)$ & $p$ \\
\hline Delayed gastric emptying & $20(39.2 \%)$ & $5(8.8 \%)$ & 0.92 \\
Grade A & $3(5.9 \%)$ & $0(0.0 \%)$ & 0.611 \\
Grade B & $6(11.8 \%)$ & $0(0.0 \%)$ & 0.48 \\
Grade C & $11(21.6 \%)$ & $5(8.8 \%)$ & 0.295 \\
Start of solid diet (day) & $4.1 \pm 1.1$ & $3.9 \pm 1.2$ & 0.277 \\
Postoperative hospital stay (day) & $22.7 \pm 24.7$ & $19.7 \pm 15.4$ & 0.353 \\
Operative time & $451.7 \pm 76.1$ & $397.7 \pm 67.2$ & 0.327 \\
Transfusion & 8 & $1(2.4 \%)$ & 1 \\
Bile leak & 1 & $0(0.0 \%)$ & 0.214 \\
Chyle leak & 6 & $0(0.0 \%)$ & 0.162 \\
Intra-abdominal fluid collection & 5 & $6(14.6 \%)$ & 1 \\
Postoperative bleeding & 1 & $1(2.4 \%)$ & 1 \\
Ileus & 2 & $1(2.4 \%)$ & 0.796 \\
POPF & & & 0.807 \\
A & 12 & $5(12.2 \%)$ & 1 \\
B & 4 & $3(7.3 \%)$ & \\
C & 0 & $0(0.0 \%)$ & 0.179 \\
Clavien-Dindo classification & & $37(90.2 \%)$ & 0.969 \\
Grade I-II & 83 & $4(9.8 \%)$ & 0.611 \\
Grade IIIa-IIIb & 5 & $0(0.0 \%)$ & $1(2.4 \%)$ \\
Grade IV-V & 1 & $2(4.9 \%)$ & \\
Reoperation & 0 & 1 & \\
30-day mortality & 0 & & \\
\hline
\end{tabular}

POPF, postoperative pancreatic fistula

( $\mathrm{n}=26$ vs. $\mathrm{n}=9, p=0.536$ ), pancreas texture (soft $\mathrm{n}=60$ vs. $\mathrm{n}=31, p=0.409$ ), and vein resection including the portal vein, superior mesenteric vein, or inferior vena cava ( $\mathrm{n}=11$ vs. $\mathrm{n}=4, p=0.908$ ) was noted between the two groups.

Table 2 shows the postoperative outcomes in both groups. The mean operative time was shorter in the SGJ than in the HGJ $(450.4 \pm 75.4 \mathrm{~min}$ vs. $397.1 \pm 66.5 \mathrm{~min}$, $p<0.001)$. The incidence of DGE in HGJ and SGJ was $12.2 \%$ and $14.6 \%$, respectively; however, there was no significant difference ( $\mathrm{n}=11$ vs. $\mathrm{n}=6, p=0.92$ ). Additionally, there were no significant differences in postoperative length of hospital stay $(22.7 \pm 24.7$ day vs. $19.7 \pm 15.4$ day, $p=0.353$ ), bile leak ( $\mathrm{n}=1$ vs. $\mathrm{n}=0, p=1)$, chyle leak $(\mathrm{n}=6$ vs. $\mathrm{n}=0, p=0.214)$, intra-abdominal fluid collection $(\mathrm{n}=5$ vs. $\mathrm{n}=6, p=0.162$ ), postoperative bleeding ( $\mathrm{n}=1$ vs. $\mathrm{n}=1$, $p=1$ ), ileus ( $\mathrm{n}=2$ vs. $\mathrm{n}=1, p=1$ ), POPF ( $\mathrm{n}=16$ vs. $\mathrm{n}=8, p=1$ ), Clavien-Dindo classification (Grade I-II $n=83$ vs. $n=37$, $p=0.969$, Grade IIIa-IIIb $\mathrm{n}=5$ vs. $\mathrm{n}=4, p=0.611$, Grade IV-V $\mathrm{n}=1$ vs. $\mathrm{n}=0, p=1)$, reoperation $(\mathrm{n}=0$ vs. $\mathrm{n}=2, p=0.179$ ), or 30 -day mortality ( $\mathrm{n}=0$ vs. $\mathrm{n}=2, p=0.179$ ) between both groups.

\section{DISCUSSION}

In this study, we compared the clinical outcomes between the stapler and handsewn anastomosis for gastrojejunostomy in patients who underwent PrPD. The operative time was significantly shorter in stapler group, while post-operative morbidity and mortality were no significantly different between the stapler and handsewn group.

There have been some attempts to use a stapler in pancreticoduodenectomy, especially to reduce the incidence of DGE in duodenojejunal anastomosis using circular stapler in PPPD. Murata et al. ${ }^{14}$ reported that the incidence of primary DGE was significantly low in the stapled side-to-side gastrojejunostomy group for subtotal stomach-preserving pancreaticoduodenectomy. This was thought to be because the stapling anastomosis, prevented anastomotic edema or stenosis, and disruption in the blood supply, and allowed easier drainage of the food contents. According to a systematic review compared to conventional handsewn anastomosis, stapled anastomosis might be associated with a lower incidence of DGE after pan- 
creaticoduodenectomy without increasing the risk of POPF or mortality. ${ }^{10,15}$ However, in some cases, the decrease in the reconstruction time was the same. A few other studies showed no difference in the incidence of DGE. ${ }^{11,16-18}$

In these studies, most of the results after gastrojejunostomy were in patients who underwent Whipple's operation or PPPD, and there were few reports about the results after gastrojejunostomy using staples in PrPD. Moreover, in most studies, several surgical methods such as Whipple's operation, PPPD, and PrPD were analyzed together. In some reports, duodenojejunostomy and gastrojejunostomy were included and analyzed together in the stapled group; hence, so there might have been some other factors influencing the DGE, such as pylorospasm. However, we analyzed only cases of PrPD for the following reasons. First, many reports have described that the rate of DGE is lower in PrPD than PPPD. ${ }^{16,19-21}$ Second, the possibility of occurrence of DGE due to the spasm of the pyloric ring was excluded by excising the pyloric ring. ${ }^{22}$ Third, excision of the pyloric ring eases the linear stapled anastomotic procedure and enables a consistent surgical method. Furthermore, single surgical method was performed by a single surgeon in order to reduce the factors that might affect the results due to the differences in the surgical methods and to limit the technical variables. Therefore, it can be presumed that the outcomes of the two groups are highly reliable.

Although this result showed that there was no difference in the rate of DGE between the groups, the use of stapler has the advantage of reducing the operation time without any difference in the incidence of other complications. Moreover, the handsewn technique might differ between each surgeon. A stapler can ensure a more standardized anastomotic method with less variation between the surgeons.

Two patients in the stapled group required re-operation. The first patient was re-operated for pancreaticojejunostomy leakage, and the second patient was re-operated for postoperative bleeding. The bleeding was not at the staple line, and thus, it might not be associated with staple use. In the HGJ group, there were no cases that required re-operation; however, one patient died. The patient had an Eastern Cooperative Oncology Group (ECOG) score of 1 due to an underlying disease and died due to postoperative pneumonia.

Our study has several limitations. First, this study was a retrospective analysis; therefore, the possibility of selection bias cannot be eliminated. In order to minimize this bias, we analyzed data for one surgical method performed by a single surgeon. Second, the different number of patients between the two groups might have affected the power of statistical evaluation. Third, when randomly checking the time of gastrojejunostomy, handsewn took 23-25 minutes and stapled was 9-10 minutes. However, the procedure time was not recorded for all patients. Fourth, this study reported only the short-term outcomes, further analysis is necessary to determine if there is any difference in the long-term outcome.

In conclusion, this study suggest that stapled GJ shortens reconstruction time during PrPD without affecting the incidence of DGE. Therefore, using a stapler for the GJ is feasible and safe technique.

\section{CONFLICT OF INTEREST}

The authors have no potential conflicts of interest to disclose.

\section{ORCID}

Sook Hyun Lee: https://orcid.org/0000-0003-3249-3914

Yun Ho Lee: https://orcid.org/0000-0001-8397-3462

Young Hoe Hur: https://orcid.org/0000-0003-1089-2033

Hee Joon Kim: https://orcid.org/0000-0002-8636-5726

Byung Gwan Choi: https://orcid.org/0000-0002-5133-1841

\section{AUTHOR CONTRIBUTIONS}

Design: YHH. Data curation: HJK, BGC. Statistical analysis: YHL. Writing - original draft: SHL. Writing review \& editing: YHH.

\section{REFERENCES}

1. Winter JM, Cameron JL, Yeo CJ, Lillemoe KD, Campbell KA, Schulick RD. Duodenojejunostomy leaks after pancreaticoduodenectomy. J Gastrointest Surg 2008;12:263-269.

2. Traverso LW, Hashimoto Y. Delayed gastric emptying: the state of the highest level of evidence. J Hepatobiliary Pancreat Surg 2008; 15:262-269.

3. Lai EC, Lau SH, Lau WY. Measures to prevent pancreatic fistula 
after pancreatoduodenectomy: a comprehensive review. Arch Surg 2009; $144: 1074-1080$.

4. Mitchell CK, Smoger SH, Pfeifer MP, Vogel RL, Pandit MK, Donnelly PJ, et al. Multivariate analysis of factors associated with postoperative pulmonary complications following general elective surgery. Arch Surg 1998;133:194-198.

5. Reich DL, Bennett-Guerrero E, Bodian CA, Hossain S, Winfree W, Krol M. Intraoperative tachycardia and hypertension are independently associated with adverse outcome in noncardiac surgery of long duration. Anesth Analg 2002;95:273-277, table of contents.

6. Nomura S, Sasako M, Katai H, Sano T, Maruyama K. Decreasing complication rates with stapled esophagojejunostomy following a learning curve. Gastric Cancer 2000;3:97-101.

7. Hyodo M, Hosoya Y, Hirashima Y, Haruta H, Kurashina K, Saito $\mathrm{S}$, et al. Minimum leakage rate $(0.5 \%)$ of stapled esophagojejunostomy with sacrifice of a small part of the jejunum after total gastrectomy in 390 consecutive patients. Dig Surg 2007;24:169-172.

8. Korolija D. The current evidence on stapled versus hand-sewn anastomoses in the digestive tract. Minim Invasive Ther Allied Technol 2008; 17:151-154.

9. Liu BW, Liu Y, Liu JR, Feng ZX. Comparison of hand-sewn and stapled anastomoses in surgeries of gastrointestinal tumors based on clinical practice of China. World J Surg Oncol 2014; 12:292.

10. Hajibandeh S, Hajibandeh S, Khan RMA, Malik S, Mansour M, Kausar A, et al. Stapled anastomosis versus hand-sewn anastomosis of gastro/duodenojejunostomy in pancreaticoduodenectomy: a systematic review and meta-analysis. Int J Surg 2017;48:1-8.

11. Sakamoto Y, Hori S, Oguro S, Arita J, Kishi Y, Nara S, et al. Delayed gastric emptying after stapled versus hand-sewn anastomosis of duodenojejunostomy in pylorus-preserving pancreaticoduodenectomy: a randomized controlled trial. J Gastrointest Surg 2016;20:595-603.

12. Wente MN, Bassi C, Dervenis C, Fingerhut A, Gouma DJ, Izbicki JR, et al. Delayed gastric emptying (DGE) after pancreatic surgery: a suggested definition by the International Study Group of Pancreatic Surgery (ISGPS). Surgery 2007;142:761768.

13. Bassi C, Marchegiani G, Dervenis C, Sarr M, Abu Hilal M, Adham M, et al. The 2016 update of the International Study
Group (ISGPS) definition and grading of postoperative pancreatic fistula: 11 years after. Surgery 2017;161:584-591.

14. Murata Y, Tanemura A, Kato H, Kuriyama N, Azumi Y, Kishiwada M, et al. Superiority of stapled side-to-side gastrojejunostomy over conventional hand-sewn end-to-side gastrojejunostomy for reducing the risk of primary delayed gastric emptying after subtotal stomach-preserving pancreaticoduodenectomy. Surg Today 2017;47:1007-1017.

15. Sakamoto Y, Yamamoto Y, Hata S, Nara S, Esaki M, Sano T, et al. Analysis of risk factors for delayed gastric emptying (DGE) after 387 pancreaticoduodenectomies with usage of 70 stapled reconstructions. J Gastrointest Surg 2011;15:1789-1797.

16. Kawai M, Tani M, Hirono S, Miyazawa M, Shimizu A, Uchiyama $\mathrm{K}$, et al. Pylorus ring resection reduces delayed gastric emptying in patients undergoing pancreatoduodenectomy: a prospective, randomized, controlled trial of pylorus-resecting versus pyloruspreserving pancreatoduodenectomy. Ann Surg 2011;253:495-501.

17. Sato N, Yabuki K, Kohi S, Mori Y, Minagawa N, Tamura T, et al. Stapled gastro/duodenojejunostomy shortens reconstruction time during pylorus-preserving pancreaticoduodenectomy. World J Gastroenterol 2013;19:9399-9404.

18. Kim DH, Hong SC, Jang JY, Cho JK, Ju YT, Lee YJ, et al. Comparing the surgical outcomes of stapled anastomosis versus hand-sewn anastomosis of duodenojejunostomy in pylorus-preserving pancreaticoduodenectomy. Ann Hepatobiliary Pancreat Surg 2019;23:245-251.

19. Huang W, Xiong JJ, Wan MH, Szatmary P, Bharucha S, Gomatos I, et al. Meta-analysis of subtotal stomach-preserving pancreaticoduodenectomy vs pylorus preserving pancreaticoduodenectomy. World J Gastroenterol 2015;21:6361-6373.

20. Zhou Y, Lin L, Wu L, Xu D, Li B. A case-matched comparison and meta-analysis comparing pylorus-resecting pancreaticoduodenectomy with pylorus-preserving pancreaticoduodenectomy for the incidence of postoperative delayed gastric emptying. HPB (Oxford) 2015;17:337-343.

21. Hanna MM, Gadde R, Allen CJ, Meizoso JP, Sleeman D, Livingstone AS, et al. Delayed gastric emptying after pancreaticoduodenectomy. J Surg Res 2016;202:380-388.

22. Kim DK, Hindenburg AA, Sharma SK, Suk CH, Gress FG, Staszewski $\mathrm{H}$, et al. Is pylorospasm a cause of delayed gastric emptying after pylorus-preserving pancreaticoduodenectomy? Ann Surg Oncol 2005;12:222-227. 